\title{
BMJ Open Effectiveness of integrated treatment for eating disorders in Spain: protocol for a multicentre, naturalistic, observational study
}

\author{
Antoni Grau Touriño (D) ," Guillem Feixas (D) ,,3 Joan Carles Medina (D) ,,5 \\ Clara Paz (iD , ${ }^{6}$ Chris Evans (iD ${ }^{7,8}$
}

To cite: Grau Touriño A, Feixas G, Medina JC, et al. Effectiveness of integrated treatment for eating disorders in Spain: protocol for a multicentre, naturalistic, observational study. BMJ Open 2021;11:e043152. doi:10.1136/ bmjopen-2020-043152

- Prepublication history for this paper is available online. To view these files, please visit the journal online (http://dx.doi. org/10.1136/bmjopen-2020043152).

Received 27 July 2020 Revised 17 February 2021 Accepted 19 February 2021

Check for updates

(C) Author(s) (or their employer(s)) 2021. Re-use permitted under CC BY-NC. No commercial re-use. See rights and permissions. Published by BMJ.

For numbered affiliations see end of article.

Correspondence to

Antoni Grau Touriño;

agrau@itasaludmental.com

\section{ABSTRACT}

Introduction Eating disorders (EDs) are complex pathologies which require equally complex treatment strategies. These strategies should be multidisciplinary, personalised interventions, performed in appropriate settings along a healthcare continuum from inpatient to community care. Personalisation, and the complexity of levels of care and interventions make evaluation of treatments difficult. The present study aims to measure the effectiveness of a complex treatment programme for EDs which includes hospitalisation, day hospital and outpatient settings. Our purpose is to assess the complete therapeutic process of each patient through all these levels of care, capturing the multiplicity of trajectories that a programme of these characteristics involves.

Methods and analysis This protocol describes a multicentre, naturalistic, observational study. All patients starting between November 2017 and October 2020 in a healthcare network for EDs in Spain are being invited to participate. The first phase of intensive change monitoring to November 2020 is followed by lower intensity followup until October 2025. In the first phase progress of all participants is assessed every 3 weeks using specific measures for ED and the Clinical Outcomes Routine Evaluation system, a family of instruments specifically designed to measure change in psychotherapy. In the second phase data collection will happen quarterly. Both cross-sectional and longitudinal analyses will be conducted, with a special focus on patterns and predictors of change studied through multilevel linear models.

Ethics and dissemination The study has been approved by the Research Bioethics Committee of the University of Barcelona (no. IRB00003099) and the ethical committee of ITA Mental Health, the organisation to which all participating centres belong. Dissemination will be in papers for peer-reviewed research journals and to clinicians working with ED.

Trial registration number NCT04127214.

\section{INTRODUCTION}

Eating disorders (EDs) are multidetermined pathologies with serious health implications. With typical onset in adolescence, they can present throughout life ${ }^{1}$ and affect approximately nine women for every man. ${ }^{2}$ Indeed,
Strengths and limitations of this study

- The present study is aimed to capture the diversity of patient's trajectories through their treatment.

- Change is measured in the context of routine clinical practice, representing the heterogeneity of eating disorders (EDs).

- The long-term longitudinal measurements allow identification of different trajectories of change among ED patients under treatment.

- The practice-based evidence approach contributes to the originality of this study, whose results will be based on real-world data.

- Heterogeneity among participants may limit generalisability of the findings and the lack of a control group precludes strong causal attributions.

prevalence reaches $12 \%$ among women aged 12-20 years. ${ }^{3}$

Anorexia nervosa (AN) and bulimia nervosa $(\mathrm{BN})$ are perhaps the best-known EDs. However, the Diagnostic and Statistical Manual of Mental Disorders 5th Edition ${ }^{4}$ also includes binge ED (BED), avoidant restrictive food intake disorder (ARFID), rumination disorders and other specified eating disorders (OSFEED).

Despite these fixed categories, two-thirds of cases experience diagnostic migration, with transition from $\mathrm{AN}$ to $\mathrm{BN}$ being the most frequent. $^{5}$ Another characteristic of EDs is the presence of comorbidities, most frequently personality, affective, anxiety and substance use disorders. ${ }^{6}$

In addition, the typical course of EDs is characterised by high rates of treatment drop-out (between $20 \%$ and $50 \%$ ), ${ }^{7}$ together with frequent relapses that may reach $51 \%$ of cases 5 years after hospital discharge. ${ }^{8}$ Unfortunately, the mortality rate for $\mathrm{AN}$ is as high as $5 \%,{ }^{8}$ and $3.9 \%$ for bulimia. ${ }^{9}$ 
The complex nature of EDs poses a challenge for the design of adequate treatment programmes. In this sense, the main clinical practice guidelines recommend treatment personalization, care continuity and the administration of interventions aimed not only to recover weight, but also to improve the associated psychological and social factors. ${ }^{1011}$ In practice, this has resulted in the design of different multicomponent treatments that combine individual, group and family interventions carried out by specialised interdisciplinary teams offering psychotherapeutic, pharmacological and nutritional support. ${ }^{12} 13$

At the same time, the inherent complexity of treating EDs has affected evaluation of outcomes. Numerous investigations in this field have studied the effects of specific psychological, pharmacological and nutritional interventions, ${ }^{1415}$ while other studies have focused on the assessment of treatment in different settings. ${ }^{12}{ }^{16-20}$ These have all produced clinical improvements, with effect sizes on ED symptoms ranging from medium to large.

However, some studies on the evaluation of results tend to limit their focus to a single disorder of the EDs spectrum (AN, BN, BED, ARFID or others), ${ }^{21}{ }^{22}$ which complicates the application of findings where there is diagnostic migration and comorbidity.

To date, very few studies have attempted to evaluate the complete therapeutic process, integrating the different types of care (eg, hospitalisation, day hospital, outpatient services). Equally, few studies have tracked use of medication. The evidence generated through such naturalistic settings is scarce, but some high-quality international studies have shown rates of positive outcome ranging between $30 \%$ and $40 \%$ at 1-year follow-up. ${ }^{23} 24$

A possible explanation for the absence of more research projects of this kind may lie in the fact that such evaluations are beyond the scope of randomised controlled trials (RCTs) which are the foundation of evidence-based practice (EBP). Although RCTs offer a gold-standard of research that has the highest internal validity for causal attributions, it is precisely this focus on internal experimental control that precludes flexibility in research design to reflect both the heterogeneity of EDs and the typical complexity of treatments. As an alternative to RCTs, several authors have proposed studies of large samples derived from routine clinical data. ${ }^{25}$ This can produce generalisable data to allow comparison between different services and can ensure inclusion and exclusion criteria that are sufficiently broad to give representative results. Such research generates so-called practice-based evidence (PBE) that focuses on broad-spectrum outcome measures applied in daily clinical practice to complement EBP. $^{26}{ }^{27}$ These measures can be used to analyse large data sets from very diverse patients with the aim of having comparable results to generate evidence on different treatments and/ or services in naturalistic settings. ${ }^{28}$

Following the PBE approach, this research programme aims to evaluate the effectiveness of an integrated treatment for EDs, specifically the ITA Model of Integrated Treatment of EDs (ITAMITED). This model prioritises care continuity through integrated hospitalisation, day hospital and outpatient services and in which each patient has personalised treatment plan. The treatment leans primarily on psychotherapy, which is conducted combining individual, group and family formats. Although not required for all patients, medication is included, targeting ED symptoms, comorbid problems or both. Given the multifactorial nature of EDs and the diversity of maintenance factors, the objectives of the interventions in the ITA model are not limited to weight improvement or other symptomatic expressions of the disorder, and instead extend to improve the discomfort created by the pathology and to promote sustainable personal aims and life style. As such, discharge criteria are not solely about remission of ED symptoms but include resolution of problems in mood, impulse control, anxiety, family relationship, affective, social and professional domains.

\section{Study objectives}

The main objective of this study is to assess the effectiveness of ITAMITED as it is applied routinely in its natural context. We aim to measure the effects of the ITA model on psychological distress, and eating attitudes and symptoms. Benchmark comparisons will be performed to estimate the relative effectiveness where suitable referential data exist in the literature. The following secondary objectives will also be pursued:

- Evaluation of the contribution of each of the intervention levels (ie, inpatient, day hospital and outpatient).

- Describe the characteristics of the study population from sociodemographic and clinical perspectives.

- Identify specific change patterns for patients with different diagnoses or individual characteristics.

- Study the psychometric properties of each instrument used in the study, the possible convergence between them and their predictive capacity regarding different clinical outcome indicators.

- Describe the main challenges in implementing a routine for measuring changes in clinical practice in a complex healthcare network.

Our main hypothesis is that the vast majority of participants will show improvements in psychological distress from baseline to treatment termination. Secondary hypotheses include improvements in secondary outcomes, and are based on the sociodemographic and clinical variables measured (see the Data analysis section). Emerging analyses might also arise in the future and they will be described as such.

\section{METHOD AND ANALYSIS \\ Study design}

This multicentre, prospective, observational naturalistic study will evaluate the effectiveness of the treatment for ED offered by ITA among new clients. All patients with a diagnosed ED admitted for treatment between November 2017 and October 2020 inclusive in any of 
the ITA treatment centres will be invited to participate. Once a participant is enrolled, they will be followed throughout the therapeutic process, within any setting in the ITAMITED treatment network, and beyond discharge through booster session contacts until October 2025 or loss of contact.

The present study is being carried out in eight EDs treatment centres of the ITA network as follows:

- Four hospitals in Barcelona, Argentona (near Barcelona), Alcalá de Henares (near Madrid) and Seville.

- Four day hospitals and outpatient care in Barcelona, Sabadell (near Barcelona), Tarragona and Madrid.

\section{Treatment}

\section{ITA and its settings}

ITA (www.itasaludmental.com) is a network of healthcare resources for the treatment for different mental disorders in Spain. Opened in 1998 with a strong emphasis on psychotherapy for EDs it has grown into an extensive network of services offering an integrated healthcare network that guarantees healthcare continuity. This continuity of care allows the patient to receive treatment according to the severity of their problems and their need for external control with high levels of coordination when care transfers between inpatient, day hospital and outpatient settings.

Inpatient hospitalisation is used to care for patients with more serious problems or with organic complications. Day hospitalisation offers treatment during the day, with patients spending the night at home, and allows patients to combine intensive therapy with studies, work or leisure activities. Finally, outpatient services have a dual purpose within ITAMITED: they are used to follow-up patients after hospital treatments but they are also the resource of choice for patients with mild symptoms who do not require day or inpatient hospitalisation.

\section{Treatment features}

\section{Therapeutic principles}

In each setting, patients are treated by a multidisciplinary team comprising a psychologist who coordinates the treatment, a psychiatrist, a general practitioner, a nutritionist, a social worker and a team of nurses and monitors. Each team shares a common view of the disorder and its therapy, adopting the ITA model.

ITAMITED adopts an integrative approach of psychotherapy. ${ }^{29}$ Cognitive, emotional and relational-systemic interventions are combined under a constructivist epistemological framework. ${ }^{30}$

\section{Psychotherapy}

During hospitalisation and day hospital stays, each patient attends three daily group therapies that are selected based on diagnosis (AN or BN), comorbidity (addiction, personality disorder), age and treatment phase. Dialectical-behavioural therapy is offered to patients with a personality disorder, and addiction therapy is offered to patients with substance abuse. Phase I of treatment is intended for newly enrolled patients and focuses on achieving awareness of the problem and encouraging motivation for change. Phase II then involves psychotherapeutic work that focuses on the core issues that appear to explain and maintain the problems. During this phase, hospitalised patients are often given agreed periods of leave to consolidate the changes in their natural environment. Patients attending day hospitals are encouraged to lessen their attendance in an agreed pattern. In both levels of hospitalisation, home stays are increased in duration as the patient's autonomy improves. Finally, phase III focuses on relapse prevention and social rehabilitation to help the patient participate again in the community through work, study and social relationships.

\section{Additional therapies}

All patients engage in two forms of family therapy: systemic therapy with their family once every 15 days and multifamily group sessions held once a month to reduce stress in the family and improve communication among its members. All patients also follow a personalised dietary programme that is formulated to recover lost weight in underweight patients, or to promote healthy weight loss in patients who are overweight or obese. Some patients may also receive pharmacotherapy for symptoms associated with EDs or for comorbid disorders.

It is well known, especially in patients with a longer duration of problems, that significant deficits can exist in the important domains of work and/or education with consequent financial challenges. Added to these difficulties, patients' social relationships are often poor and their social networks restricted. Reflecting these challenges, all patients follow a psychosocial rehabilitation programme in which a team of social educators, social workers and educational psychologists work with them on these areas. The socioeducational programme also ensures that patients of school-age continue with their education as normally as possible, either within the hospital or adapting the treatment to allow attendance at their usual school.

\section{Participants}

All patients who start treatment for EDs at ITA between 2 November 2017 and 2 October 2020, are invited to participate in this study.

\section{Inclusion and exclusion criteria}

Inclusion criteria

- A diagnosis of ED: AN, BN, BED, ARFID, OSFEED or ED not specified.

- To follow treatment for ED in one of the ITA centres.

\section{Exclusion criteria}

- Refusal to participate (or)

- Inability to give valid consent or to understand the questionnaires due to language, visual or cognitive barriers.

At admission, all patients are informed about the study and are offered the option to participate, agreement 


\begin{tabular}{|c|c|c|c|c|c|c|c|c|c|}
\hline TIMEPOINT & & 0 & W1 & $W 2$ & M3 & M6 & M9 & M12 & Discharge \\
\hline \multirow{4}{*}{$\begin{array}{l}\text { FIRST } \\
\text { CONTACT } \\
\text { WITH ITA }\end{array}$} & \multirow{4}{*}{$\begin{array}{l}\text { Informed consent } \\
\text { Sociodemographic } \\
\text { information } \\
\text { Clinical information } \\
\text { Life satisfaction scale }\end{array}$} & $x$ & & & & & & & \\
\hline & & $x$ & & & & & & $x$ & \\
\hline & & $x$ & & & & & & $x$ & \\
\hline & & $x$ & & & & & & $x$ & \\
\hline \multirow[t]{5}{*}{ ASSESSMENT } & $\begin{array}{l}\text { CORE-OM ( } \geq 18 \text { years) } \\
\text { YP-CORE (<18 years) }\end{array}$ & & $x$ & $x$ & $x$ & $x$ & $x$ & $x$ & $x$ \\
\hline & $\begin{array}{l}\text { EAT-26 ( } \geq 18 \text { years) } \\
\text { CHEAT-26 (<18 years) }\end{array}$ & & $x$ & $x$ & $x$ & $x$ & $x$ & $x$ & $x$ \\
\hline & BITE & & $x$ & $x$ & $x$ & $x$ & $x$ & $x$ & $x$ \\
\hline & \multirow{2}{*}{$\begin{array}{l}\text { CORE-SFA/SFB ( } \geq 18 \text { years) } \\
\text { YP-CORE }(<18 \text { years) }\end{array}$} & & & & \multicolumn{4}{|c|}{$\begin{array}{l}\text { Hospitalization or day } \\
\text { hospital } \\
\text { Every three weeks }\end{array}$} & \\
\hline & & & & & \multicolumn{4}{|c|}{$\begin{array}{l}\text { Outpatient } \\
\text { Every session }\end{array}$} & \\
\hline
\end{tabular}

Figure 1 Schedule for data collection and assessments for a 12-month treatment. This table represents an example of data collection and assessment for patients within the service for 1 year. Data collection beyond that point continues as it was in the first year until the termination of intensive data collection in October 2020. From November 2020 to end of October 2025, CORE-OM and EAT-26 (or ChEAT-26) will be administered every 3 months. BITE, Bulimic Investigatory Test of Edinburgh; ChEAT-26, Children's Eating Attitudes Test; CORE-OM, Clinical Outcomes in Routine Evaluation-Outcome Measure; CORE-SFA, Clinical Outcome in Routine Evaluation Short Form A; CORE-SFB, Clinical Outcome in Routine Evaluation Short Form B; EAT26, Eating Attitudes Test; W1/2, Week 1/2; M3/6/9/12, month 3/6/9/12 from time 0; YP-CORE, Young Person's CORE.

is recorded if the patient chooses to sign the informed consent form. Participation is voluntary and confidential. For those younger than 18 years, consent must also be signed by an appropriate relative or legal guardian.

\section{Measures}

Primary change measures

Clinical Outcomes in Routine Evaluation system

The Clinical Outcomes in Routine Evaluation (CORE) system is a family of instruments designed to measure change in psychological distress occurring in the context of psychotherapy within a transdiagnostic and pantheoretical framework (www.coresystemtrust.org.uk). Questionnaires have been translated into more than 25 languages and the measures are widely used internationally to monitoring change in many services, languages and countries. From this family of measures, we included the following forms according to participant characteristics and the assessment point (see figure 1).

CORE-Outcome Measure (CORE-OM). The main questionnaire consists of 34 items divided across four subdomains: subjective well-being, problems or symptoms, general functioning and risk. It is suitable for adults and has shown good psychometric properties in evaluations of the original English (Cronbach $\alpha=0.75-0.94)$ and the Spanish $(\alpha=0.71-0.94)$ versions in samples from the UK and Spain. ${ }^{31-33}$

CORE Short Form (CORE-SFA/CORE-SFB). These short versions of CORE consist of 18 items each taken from among the 34 items of the CORE-OM and are administered alternately to minimise memory effects. Due to the item selection, they are comparable and offer scores on the same subdomains as the CORE$\mathrm{OM},{ }^{31}{ }^{32}$ showing internal consistency $(\alpha=0.94) .{ }^{34}$

Young Person's CORE (YP-CORE). This questionnaire was originally developed for use in people aged 11-16 years and comprises 10 items with results given on a single global scale. It is widely used for young people up to the age of 25 in many services. Both the original $(\alpha=0.73-0.80)^{3536}$ and the Spanish version $(\alpha=0.72-0.86)^{37}$ have shown good psychometric properties.

\section{Secondary outcomes}

Eating Attitudes Test and Children's Eating Attitudes Test

The Eating Attitudes Test (EAT-26) is a self-completed 26-item scale that is intended to assess the risk of having an ED. ${ }^{38}$ Each item has six response options and they are scored on three scales: diet, BN and concern with food. Although intended as a screening instrument it has been widely used as a severity index. For patients under 16 the Children's Eating Attitudes Test (ChEAT) ${ }^{40-42}$ replaced the EAT-26. This is similar to EAT-26 but written with simpler words. Both instruments are widely used and have shown good psychometric properties (EAT, English $\alpha=0.90$, Spanish $\alpha=0.86$; ChEAT, English $\alpha=0.76$, Spanish $\alpha=0.86)$.

\section{Bulimic Investigatory Test of Edinburgh}

This 33-item questionnaire was designed for use in BN and $\mathrm{BED}^{43}$ and generates scores for two subscales: eating symptoms and severity. Both the original $(\alpha=0.62-0.96)$ and the Spanish $(\alpha=0.63-0.82)^{44}$ versions have shown good psychometric properties. In our study, we used the 
Bulimic Investigatory Test of Edinburgh (BITE) for all patients regardless of diagnosis with the aim of better capturing the presence of binges and other abnormal eating behaviours in patients with AN.

\section{Body Mass Index}

The Body Mass Index (BMI) is a standardised indicator that results from dividing weight in kilograms by height in metres and is one of the most widely used indices of therapeutic weight change in outcome studies of ED and obesity programmes. In this study, patients' weight and height is recorded at baseline and then weight every month until the end of treatment. If treatment is still in course after 30 October 2020, weight will be recorded every 3 months until the end of treatment.

\section{Life Satisfaction Scale}

This is a 10-point Likert-type single-item scale that asks patients to rate the degree to which they are satisfied with life at the present time. Although multicomponent scales exist that can measure this construct, simple single-item scales like this are used in many population studies. ${ }^{45}$ This format was chosen for its efficiency and its theoretical consistency, which has been suggested to be greater than other more complex formats, ${ }^{46}$ while retaining an acceptable reliability $(\alpha=0.61-0.74) .{ }^{47}$

\section{Procedure}

\section{Assessment}

Participants completed the questionnaires on paper. When a new client arrives at any of the centres the receptionist gives the client (and parents if the client is under age) copies of the information sheet, consent form and initial measures, these explain that the study is voluntary and should be discussed with the assessing clinicians. The study is then explained orally by the assessing clinician, complementing the information on paper and any questions answered. Where clients agree to participate, they then filled in the questionnaires on paper. The data were checked, weight and height measured and diagnosis and medication data collected by the primary clinician during the first weeks of assessment.

An overview of the schedule is provided in figure 1. Type of input (inpatient, day hospital or outpatient: 'intensity') and other details of the services patients receive throughout their treatment in the ITAMITED system are also recorded and entered into the database.

\section{Patients who follow treatment in hospitalisation or day hospital}

During the first week, the CORE-OM (or YP-CORE) and EAT-26 (or ChEAT-26) questionnaires (depending on age) are administered, together with the BITE questionnaire. This assessment is repeated during the second week to reveal any abrupt change secondary to admission. The ChEAT-26 and YP-CORE are then administered every 3 weeks for participants under the age of 16 and the EAT-26 with either the CORE-SFA and CORE-SFB (alternated) on the same three weekly schedule for adults. The full CORE-OM (or YP-CORE),
EAT-26 (or ChEAT-26) and BITE questionnaires are administered again every 3 months. When the participant is discharged from any given level of intervention, the CORE-OM (or YP-CORE), EAT-26 (or ChEAT-26) and the BITE questionnaires are administered at the last session of treatment.

\section{Outpatient treatment}

Questionnaires are administered by administrative staff just before consultation with the psychotherapist. The CORE-OM (or YP-CORE), EAT-26 (or ChEAT-26) and BITE questionnaires are administered on the first and second visits. Again, the YP-CORE is administered for participants younger than 16 years and the CORE-SFA and CORE-SFB are alternated for adults. The CORE-OM (or YP-CORE), EAT-26 (or ChEAT-26) and BITE questionnaires are then administered every 3 months, and the CORE-OM (or YP-CORE) questionnaire is administered again at discharge.

\section{For all participants}

After the end of October 2020 all participants still in treatment move to a much less intensive data collection. In this phase only the CORE-OM (or YP-CORE) and EAT-26 (or ChEAT-26) are administered approximately every 3 months until the end of October 2025. Weight is recorded as determined clinically. Pharmacological treatment, current diagnosis and days of different types of care are extracted from the services routine database. Every 12 months, each participant answers the life satisfaction scale and the sociodemographic data are recorded again to reflect changes (if any) in variables such as marital status or occupation.

\section{Data processing}

Technical issues with the services' routine data systems mean that data collection in the intensive phase could not be achieved from those systems. In this phase, each of the eight participating treatment centres entered data into its own Excel spreadsheet and a psychologist oversees data entry three times per week. Each centre shared their spreadsheets weekly with the primary coordinating centre where one clinician checked the data again and removed identifying information other than a research ID code. The pseudonymised spreadsheets were then shared with the research centre in UB where they were analysed for coherence and data entry problems using an R script. ${ }^{48}$ This analysis produced a weekly feedback report for each clinical centre noting any omissions or possible or definite errors in the data. A possible error might be a surprisingly large change in weight, a definite error might be a discharge date in the future or before the admission date. In addition to flagging possible and definite errors the report alerted each centre to the nearest dates for review data to be entered: annual for demographic data; monthly for weight, height and service use; three weekly for questionnaires; and quarterly for medication and diagnoses. 
To guarantee confidentiality, the coordinator ensures that all personal information is removed from the database before it is sent to a member of the team at the University of Barcelona so that only the professionals directly responsible for patient care within ITA can map from study ID codes to clinical ID codes. Data analysis and interpretation will always protect against identification. All storage and transfers are secure comply with the European data protection law (2016/679).

All personal information collected for this study will be stored at the ITA centres for a minimum period of 5 years after the end of the study to ensure rights of access, rectification, cancellation, limitation of treatment and opposition of the participants. However, the fully anonymised data may be stored indefinitely for future research and analysis. Options will be explored for releasing the dataset to the scientific community in an open repository if we can ensure safe use that is compliant with the General Data Protection Regulation and immune against ‘jigsaw' de-anonymisation.

\section{Data analysis}

\section{Overview}

The ITAMITED programme is highly complex and personalised, allowing different patients to take very different treatment trajectories, such as transfers between intervention types, discharge and re-entry, as well as differences in psychotropic medication use. In addition, patients have a range of primary ED and comorbidities, and they have substantial sociodemographic variety. There are generally few male patients but a wide age range at first contact. This reflects the realities of clinical work with patients who often have long histories of problems and (not infrequently) life-threatening states. These issues present unique challenges for data analysis many of which simply cannot arise in analyses of controlled trial data. The analyses will be based on three tranches of data defined by the entry cohorts and by whether the focus is on baseline characteristics (and psychometrics) or on treatment trajectories and change patterns. Very diverse methods of analysis of such complex data are available and there is none of the (relative) clarity of analysis of an RCT. It was agreed in the creation of the study that data analysis in the first 2 years would be restricted to simple analysis of baseline psychometric (allowing for removal of measures showing unacceptable psychometrics) and to description of the participants and baseline/discharge change at 1 year and 2 years. These have so far only been shared at conferences and inside ITA. A more extensive data analysis plan (DAP) is being registered with the CERN Zenodo system and will notarise emergent analyses.

\section{Analysis timing}

\section{First data tranche}

This will focus on the baseline data for participants recruited from November 2017 to October 2020. The most important focus will be to establish the baseline distributions of variables that will be used later to measure change (ie, BMI, CORE, EAT-26/ChEAT-26 and BITE). We will also report the psychometric properties of the self-report measures including acceptability, possible floor and/or ceiling effects for items, internal reliability (Cronbach alpha and McDonald Omega) of scores and discriminant and convergent validity. Finally, we will explore any differences between centres and any associations between demographic and clinical variables, such as diagnosis or medication.

\section{Second data tranche}

This will contain all data collected by the end of October 2020 (new patients entering the service during October 2020 will not be invited into the study as they would only have the first two data collection points by the time intensive change measures stop). This second data tranche will have from 1 month to 3 years of intensive change measurement. When analysing these data, the focus will be on diversity of interventions and of change, but specifically the following:

- Patterns of change in key variables (ie, BMI, CORE, EAT-26/ChEAT-26 and BITE).

- Predictors of change/outcome, including any differences between services.

- Changes in sociodemographic and clinical intervention variables (ie, diagnosis, medication or hospitalisation status).

\section{Third tranche: continuing re-assessment data}

To strike a balance between the financial and logistic challenges of intensive change data collection and the often chronic nature of EDs from the beginning of October 2020 no new patients will be invited into the study and from the beginning of November 2020 data collection will switch to collect questionnaire data only every 3 months with the addition of information about weight (and height for younger participants), diagnoses, medication, days in different types of care from the new ITA electronic data system). This data collection will be restarted (assuming continuing informed consent) for any participants returning to the service after discharge in this second period of up to 5 years, that is to the end of October 2025.

\section{Cohort size}

- The plan (see registration) aimed for a total cohort of 800 to be recruited by the end of October 2020. This was achieved with 942 currently recruited.

\section{Specific analyses}

\section{Effectiveness}

These all address change on the primary and secondary outcome variables (see above) and three approaches are used.

- Baseline/termination change reporting mean change with effect sizes (Cohen's d2 and dz), both with bootstrapped 95\% CIs. These analyses provide the most general comparability with existing effectiveness findings about ED treatments. 
- Multilevel models (MLM) of rates of change. These analyses are increasingly replacing baseline/termination analyses and analyse trajectories of change based on all repeated measures as opposed to just the first and last measures.

- Survival analysis by time to reaching scores below clinical cutting points. These methods address the inevitable 'right censoring' of data in naturalistic data sets, that is, that later recruited participants will generally have shorter durations of repeated assessment and in which participants opt out of either treatment or just of research participation, at varying points in their potential assessment time.

Predictor/subgroup analyses will address gender, age (within the age ranges determine by the measures, that is, above or below 18 years for the primary outcome CORE measures and above and below 16 years for the EAT-26/ CHEAT secondary measure), specific ED diagnosis, presence of a personality disorder secondary diagnosis.

Additional analyses will provide context to these effectiveness findings. These will be in four groups:

- Careful description of the participants including baseline demographics and medication.

- Analyses of the psychometric properties of all measures.

- Description of the diversity of change trajectories (with attention to the predictors/subgroups noted earlier).

- Description of diverse trajectories of participants including any opting out of therapy and returns, medication changes and transitions between levels of care.

All reporting will respect the above a priori designation of primary and secondary outcome variables and of predictor/subgroup planned contrasts. Emergent findings will be clearly designated as such. The publicly available, notarised DAP will detail any post hoc analyses arising either to describe emergent findings other than the a priori ones detailed above or arising through development of new statistical methods.

\section{Patient and public involvement}

Patients or the public were not involved in the design, conduct, reporting or dissemination plans of our research.

\section{DISCUSSION}

The main objective of this study is to appraise the effectiveness of a treatment model designed to deal with the complexity and heterogeneity of EDs. This model includes personalised treatments at different levels of care administered by interdisciplinary teams. Moreover, it also features a multicomponent programme consisting of individual and group psychotherapy, integrated with pharmacological and nutritional treatment. By pursuing this goal, we seek to contribute to fill the gap in PBE research in EDs, especially conducted in private institutions. ${ }^{49}$ This approach will help us overcome the artificial segmentation that occurs when evaluating healthcare processes in isolation.

The comprehensive data collection will support not only the general effectiveness analyses (see above) but allows identification of different trajectories through the care levels and the findings should help predict individual treatment outcomes and tailor interventions to personal client characteristics.

The naturalistic nature of this study will allow the inclusion of the different ED diagnoses as well as their comorbid pathologies. In effect, the collection of routine clinical data will contribute to PBE, offering results that are more directly applicable to routine practice than that from controlled trials. In this sense, the characteristics described for our study sample will enable other researchers to compare our findings with their own. Also, the psychometric properties estimated for the instruments employed will provide further information to assess their performance and utility in clinical settings.

The devised dissemination strategy is anchored in publication of results in optimal peer-reviewed research journals. However, being aware of the current practice/ research gap, we also aim to disseminate findings more directly to professionals involved in the treatment of EDs. Such actions will seek to promote engagement in collaborative PBE networks allowing routine change data to fuel the search for effective and efficient interventions.

A major challenge of this study will be to make routine data from clinical practice useful for measuring specific outcomes. Participants are being invited to complete questionnaires frequently, moving beyond the traditional approach of taking measurements before and after treatment. We believe this step is necessary to capture further complexities of change and of the varied treatment trajectories that may establish between those time points. Moreover, beyond the results we finally achieve, this study also wants to contribute to give a greater role to patients, listening to their own evaluations of their state in a systematic way. With the future reporting of difficulties and facilitators encountered in the conduction of this project we hope to spark the interest of more practitioners and researchers in monitoring change in their services. As an example, during the COVID-19 outbreak in 2020, physical distancing regulations led to interventions at day hospitals and outpatient services moved online, as were all questionnaires. To deal with this situation was a valuable opportunity to adapt PBE not only to routine challenges, but also to emergent ones.

\section{Study limitations}

Complexities occur when so many variables are considered and each participant has a personalised and regular review of their clinical treatment plan. This means that estimation of population effects (ie, of possible generalisability of findings) inevitably has wide confidence intervals for smaller cell sizes. Examples include the less common diagnoses; considering both primary and secondary diagnoses; analyses by gender 
and age groups in demographic terms. Likewise, any estimation of the influence of social variables (eg, social status, education, employment and parenting) is bound to have wide intervals. Consequently, disentangling for instance the effects of different intervention levels from other predictors can only ever be tentative since changes in the level of care are likely to be in response to clinical state and risk.

Such PBE work requires collaboration between clinical and administrative staff members, and patients, for a long time. As in any study, loss of data arises from patients opting out, either at baseline or at any point in the repeated-measures data collection. In the latter case, the removal of earlier data may be required by some participants, while others may give their consent to keep their previous information but opt out for the remaining study duration. Yet others may simply refuse participation on one occasion but participate again on later ones. Similarly, professional or administrative errors may become another source of data loss, while sometimes asking patients to complete the self-report measures will be deemed inappropriate according to clinical judgement (eg, when a patient is in a state of agitation). Such data will not be missed at random, so appropriate efforts will need to be made to describe why they were missing and the impact they had on our findings. We are sceptical about claims that any methods for imputing missing values can be unbiased but such methods will be used for sensitivity analyses to explore the possible impacts of data losses.

Finally, this study measures only a few of the many plausible variables pertinent to change in EDs: quality social support, personal histories or motivation to change. Taking into account the numerous and systematic assessments, authors have preferred to focus on some outcomes considered central to monitor change, and to only address predictors arising from demographic and clinical intervention levels. Clearly, other longitudinal studies evaluating the concomitant aspects frequently associated to EDs are required in the future.

\section{ETHICS AND DISSEMINATION}

The study has been approved by the Research Bioethics Committee of the University of Barcelona (no. IRB00003099) and the ethical committee of ITA Mental Health, who is the organisation to which all participating centres belong. Dissemination will be in papers for peerreviewed research journals but then to clinicians working with ED. Research analyses will be in three tranches: baseline analyses and psychometrics of measures; detailed change patterns and service trajectories; follow-up: results of the study will also be presented in national and international conferences. All dissemination will be compliant with the Strengthening the Reporting of Observational Studies in Epidemiology/Declaration of Altman and Moher of transparency. ${ }^{50}$

\section{Author affiliations}

${ }^{1}$ Clinical Management, ITA Mental Health, Barcelona, Spain

2Department of Clinical Psychology and Psychobiology, Section of Personality,

Evaluation and Psychological Treatment, Psychology Faculty, Universitat de

Barcelona, Barcelona, Spain

${ }^{3}$ The Institute of Neurosciences, Universitat de Barcelona, Barcelona, Spain ${ }^{4}$ Department of Clinical Psychology and Psychobiology, Universitat de Barcelona, Barcelona, Spain

${ }^{5}$ Faculty of Psychology and Education Sciences, Universitat Oberta de Catalunya, Barcelona, Spain

${ }^{6}$ School of Psychology, Universidad de Las Americas, Quito, Ecuador ${ }^{7}$ Department of Psychology, The University of Sheffield, Sheffield, UK

${ }^{8}$ Department of Psychology, University of Roehampton, London, UK

Contributors AGT, GF, JCM and CE conceptualised the overall project and colead the design of the intensive phase of the study. AGT, CE coled the design of the follow-up phase. AGT, JCM and CE contributed to the plan of analyses. CE led the weekly data quality monitoring. JCM contributed to the quality of data monitoring. AGT drafted the manuscript. GF, JCM, CP and CE edited and reviewed the manuscript. All authors read and approved the final manuscript.

Funding This work was supported by ITA Mental Health with a grant number FBG309562.

Competing interests AGT is the Clinical Director at ITA Mental Health, the network financing the study and in which it is being conducted. ITA was motivated to explore the effectiveness and any differential effectiveness of the treatment programme by centre, hence, funded data collection within ITA and also funded an external organisation, University of Barcelona, to deliver scientific advice in the design and implementation of data collection, to ensure database integrity, and to ensure independent analysis. The data collection has generated a pseudonymised dataset which is held by UB and CE. ITA Mental Health and the University of Barcelona (team led by GF) have established a contract for this collaboration (ref. FBG309562). GF is the Principal Investigator of the research group involved in this contract.

Patient and public involvement Patients and/or the public were not involved in the design, or conduct, or reporting, or dissemination plans of this research.

Patient consent for publication Not required.

Provenance and peer review Not commissioned; externally peer reviewed.

Open access This is an open access article distributed in accordance with the Creative Commons Attribution Non Commercial (CC BY-NC 4.0) license, which permits others to distribute, remix, adapt, build upon this work non-commercially, and license their derivative works on different terms, provided the original work is properly cited, appropriate credit is given, any changes made indicated, and the use is non-commercial. See: http://creativecommons.org/licenses/by-nc/4.0/.

\section{ORCID iDs}

Antoni Grau Touriño http://orcid.org/0000-0003-0745-653X

Guillem Feixas http://orcid.org/0000-0002-6288-6009

Joan Carles Medina http://orcid.org/0000-0002-4550-2157

Clara Paz http://orcid.org/0000-0003-1106-9567

Chris Evans http://orcid.org/0000-0002-4197-4202

\section{REFERENCES}

1 Mangweth-Matzek B, Hoek HW. Epidemiology and treatment of eating disorders in men and women of middle and older age. Curr Opin Psychiatry 2017;30:446-51.

2 Striegel-Moore RH, Bulik CM. Risk factors for eating disorders. Am Psychol 2007;62:181-98.

3 Stice E, Marti CN, Shaw H, et al. An 8-year longitudinal study of the natural history of threshold, subthreshold, and partial eating disorders from a community sample of adolescents. J Abnorm Psychol 2009;118:587-97.

4 American Psychiatric Association. Diagnostic and statistical manual of mental disorders. 5th edn. American Psychiatric Association, 2013.

5 Fairburn CG, Cooper Z, Doll HA, et al. Transdiagnostic cognitivebehavioral therapy for patients with eating disorders: a two-site trial with 60-week follow-up. Am J Psychiatry 2009;166:311-9.

6 Hudson JI, Hiripi E, Pope HG, et al. The prevalence and correlates of eating disorders in the National comorbidity survey replication. Biol Psychiatry 2007;61:348-58. 
7 Dejong $\mathrm{H}$, Broadbent $\mathrm{H}$, Schmidt U. A systematic review of dropout from treatment in outpatients with anorexia nervosa. Int J Eat Disord 2012;45:635-47.

8 Huas C, Godart N, Caille A, et al. Mortality and its predictors in severe Bulimia nervosa patients. Eur Eat Disord Rev 2013;21:15-19.

9 Van Elburg A. Psychoneuroendocrinological aspects of anorexia nervosa: predictors of recovery, 2007.

10 National Institute for Health and Care Excellence. Eating disorders: recognition and treatment, 2017. Available: https://www.nice.org. uk/guidance/ng69/resources/eating-disorders-recognition-andtreatment-pdf-1837582159813

11 American Psychiatric Association. Treatment of patients with eating disorders, third edition. American Psychiatric Association. Am J Psychiatry 2006;163:4-54.

12 Thompson-Brenner $\mathrm{H}$, Boswell JF, Espel-Huynh $\mathrm{H}$, et al. Implementation of transdiagnostic treatment for emotional disorders in residential eating disorder programs: a preliminary pre-post evaluation. Psychother Res 2019;29:1045-61.

13 Weltzin T, Kay B, Cornella-Carlson T, et al. Long-Term effects of a multidisciplinary residential treatment model on improvements of symptoms and weight in adolescents with eating disorders. J Groups Addict Recover 2014;9:71-85.

14 Brauhardt A, de Zwaan M, Hilbert A. The therapeutic process in psychological treatments for eating disorders: a systematic review. Int J Eat Disord 2014;47:565-84.

15 Davis H, Attia E. Pharmacotherapy of eating disorders. Curr Opin Psychiatry 2017;30:452-7.

16 Abbate-Daga G, Marzola E, De-Bacco C, et al. Day hospital treatment for anorexia nervosa: a 12-month follow-up study. Eur Eat Disord Rev 2015;23:390-8.

17 Friedman K, Ramirez AL, Murray SB, et al. A narrative review of outcome studies for residential and partial hospital-based treatment of eating disorders. Eur Eat Disord Rev 2016;24:263-76.

18 Zanna V, Castiglioni MC, Criscuolo M, et al. Day-Hospital multifocal Integrated treatment for anorexia nervosa in adolescents: a one-year follow-up. J Child Fam Stud 2017;26:1460-71.

19 Brown TA, Cusack A, Anderson LK, et al. Efficacy of a partial Hospital programme for adults with eating disorders. Eur Eat Disord Rev 2018;26:241-52.

20 Simic M, Stewart CS, Eisler I, et al. Intensive treatment program (ITP): a case series service evaluation of the effectiveness of day patient treatment for adolescents with a restrictive eating disorder. Int $J$ Eat Disord 2018;51:1261-9.

21 Sproch LE, Anderson KP, Sherman MF, et al. A randomized controlled trial of group cognitive remediation therapy for anorexia nervosa: effects on set-shifting tasks for inpatient adults and adolescents. Int J Eat Disord 2019;52:1004-14.

22 Brockmeyer T, Friederich H-C, Küppers C, et al. Approach bias modification training in Bulimia nervosa and binge-eating disorder: a pilot randomized controlled trial. Int J Eat Disord 2019;52:520-9.

23 Kordy H, for COST Action B6. Counting the cost: a European collaboration on the efficiency of psychotherapeutic treatment of patients with eating disorders. Eur Eat Disorders Rev 2005;13:153-8.

24 Richard M, Kordy H, for COST Action B6. Effective treatment of eating disorders in Europe: treatment outcome and its predictors. Eur. Eat. Disorders Rev. 2005;13:169-79.

25 Ammerman A, Smith TW, Calancie L. Practice-Based evidence in public health: improving reach, relevance, and results. Annu Rev Public Health 2014;35:47-63.

26 Margison FR, Barkham M, Evans C, et al. Measurement and psychotherapy. evidence-based practice and practice-based evidence. Br J Psychiatry 2000;177:123-30.

27 Barkham M, Stiles WB, Lambert MJ. Building a Rigorous and Relevant Knowledge Base for the Psychological Therapies. In: Developing and delivering practice-based evidence. Chichester, UK: John Wiley \& Sons, Ltd, 2010: 21-61.

28 Barkham M, Delgadillo J, Firth N. Practice based evidence and the law of variability in psicological treatment. Rev Argentina Clínica Psicológica 2018;27:115-25.
29 Feixas G, Botella L. Psychotherapy integration: reflections and contributions from a constructivist epistemology. J Psychother Integr 2004;14:192-222.

30 Feixas G. Personal constructs in systemic practice. In: Neimeyer R, Mahoney MJ, eds. Constructivism in psychotherapy. Washington, DC, US: American Psychological Association, 1995: 305-37.

31 Evans C, Mellor-Clark J, Margison F, et al. Core: clinical outcomes in routine evaluation. J Ment Heal 2000;9:247-55

32 Feixas G, Evans C, Trujillo A. La versión española del CORE-OM : Clinical Outcomes in Routine Evaluation - Outcome Measure. Rev Psicoter 2012;23:109-35.

33 Trujillo A, Feixas G, Bados A, et al. Psychometric properties of the Spanish version of the Clinical Outcomes in Routine Evaluation Outcome Measure. Neuropsychiatr Dis Treat 2016;12:1457-66.

34 Barkham M, Margison F, Leach C, et al. Service profiling and outcomes benchmarking using the CORE-OM: toward practicebased evidence in the psychological therapies. $J$ Consult Clin Psychol 2001;69:184-96.

35 Twigg E, Cooper M, Evans C, et al. Acceptability, reliability, referential distributions and sensitivity to change in the young person's clinical outcomes in routine evaluation (YP-CORE) outcome measure: replication and refinement. Child Adolesc Ment Health 2016;21:115-23.

36 Twigg E, Barkham M, Bewick BM, et al. The Young Person's CORE: Development of a brief outcome measure for young people. Couns Psychother Res 2009:9:160-8.

37 Feixas G, Badia E, Bados A, et al. Adaptation and psychometric properties of the Spanish version of the yp-core (young person's clinical outcomes in routine evaluation). Actas Esp Psiquiatr 2018;46:75-82.

38 Garner DM, Olmsted MP, Bohr Y, et al. The eating attitudes test: psychometric features and clinical correlates. Psychol Med 1982;12:871-8

39 Gandarillas A, Zorrilla B, Sepúlveda A. Trastornos del comportamiento alimentario: Prevalencia de casos clínicos en mujeres adolescentes de la Comunidad de Madrid. Madrid, Spain, 2003.

40 Maloney MJ, McGuire J, Daniels SR, et al. Dieting behavior and eating attitudes in children. Pediatrics 1989;84:482-9.

41 Rojo-Moreno L, García-Miralles I, Plumed J, et al. Children's eating attitudes test: validation in a sample of Spanish schoolchildren. Int $J$ Eat Disord 2011:44:540-6.

42 Maloney MJ, McGuire JB, Daniels SR. Reliability testing of a children's version of the eating attitude test. $J$ Am Acad Child Adolesc Psychiatry 1988;27:541-3.

43 Henderson M, Freeman CP. A self-rating scale for bulimia. The 'BITE'. Br J Psychiatry 1987;150:18-24.

44 Rivas-Moya MT, Jiménez M, Bersabé-Morán R. Fiabilidad Y validez del test de Investigación Bulímica de Edimburgo (bite) en Una muestra de adolescentes españoles. Behav Psychol Conduct 2004;12.

45 Carr A. Positive psychology: the science of Happiness and human strengths. 2nd ed. New York, NY: Routledge, 2011.

46 Cummins R. Measuring happiness and subjective well-being. In: David SA, Boniwell I, Conley Ayers A, eds. Oxford Handbook of Happiness. Oxford, UK: Oxford University Press, 2013: 185-200.

47 Lucas RE, Donnellan MB, Brent Donnellan M. Estimating the reliability of single-item life satisfaction measures: results from four national panel studies. Soc Indic Res 2012;105:323-31.

48 R Core Team. R: a language and environment for statistical computing, 2019.

49 Attia E, Marcus MD, Walsh BT, et al. The need for consistent outcome measures in eating disorder treatment programs: a proposal for the field. Int J Eat Disord 2017;50:231-4.

50 Altman DG, Moher D. Declaration of transparency for each research article. BMJ 2013:347:f4796 